\title{
A novel microcrystalline tyrosine- adsorbed, mite-allergoid subcutaneous immunotherapy: 1-year follow-up report
}

\begin{abstract}
Aim: A 1-year follow-up study comparing the safety and tolerability of the dosing schedules, satisfaction and effectiveness of a novel microcrystalline tyrosine-adsorbed mite (Dermatophagoides pteronyssinus)-allergoid subcutaneous immunotherapy (Acarovac Plus ${ }^{\mathrm{TM}}$ ) in 30 adult patients (18-65 years) with allergic rhinitis and/or asthma. Materials \& methods: The effectiveness of the product was assessed by nasal provocation test measuring peak nasal inspiratory flow/symptoms, in vitro immunologic changes (IgE, IgG4 and IL-10) and Treatment Satisfaction Questionnaire for Medication. Results: No adverse events were reported during dosing schedules. Significant decreases in symptom scores and drop of peak nasal inspiratory flow in follow-up visits (4 weeks and 1 year) were recorded. Significant increases in IgG4specific antibody titers and IL-10 were exhibited. Conclusion: Significant decreases in clinical symptoms and immunological parameters were observed, accompanying a high level of patient satisfaction and tolerance.
\end{abstract}

First draft submitted: 17 May 2016; Accepted for publication: 21 June 2016;

Published online: 7 July 2016

Keywords: Acarovac Plus ${ }^{\mathrm{TM}} \bullet$ allergen $\bullet$ allergic rhinitis $\bullet$ allergoid $\bullet$ cluster $\bullet$ conventional

- Dermatophagoides pteronyssinus $\bullet$ immunotherapy $\bullet$ nasal challenge test $\bullet$ subcutaneous

House dust mite allergy is a burgeoning condition with a substantial increase in prevalence worldwide over the last three decades, causing rhinoconjunctivitis and/or asthma [1,2]. A clinical study was conducted in the Allergy Unit of Germans Trias i Pujol University Hospital (Badalona, Spain) in December 2012 and finished in April 2013. The investigation was a prospective, observational, postauthorization study of the safety, tolerability and effectiveness of a novel microcrystalline tyrosineadsorbed, modified allergen immunotherapy (Acarovac Plus ${ }^{\mathrm{TM}}$ ) with extract of Dermatophagoides pteronyssinus in adult patients with allergic rhinitis and/or asthma [3]. The product platform is based upon the concept of modified allergen preparations (i.e., allergoids) after treatment with glutaraldehyde. The formation of allergoids in this way results in the disruption of the $3 \mathrm{D}$ structure of the proteins and its conformational IgE epitopes, thus reducing allergenicity [4]. This is achieved through extensive crosslinking between primary amines of polypeptide chains, resulting in high-molecular-weight allergoid complexes. However, retention of contiguous IgG (T-cell) epitopes maintains immunogenicity of the active substance [4]. The resulting preparation is adsorbed to L-Tyrosine (microcrystalline tyrosine [MCT] ), a patented depot adjuvant formulation. Aluminium hydroxide or aluminium phosphate are most commonly used as adjuvants for vaccines to enhance the immune response, however, an alternative biodegradable depot adjuvant formulation was sought that has been shown to support the immunological response in specific immunotherapy, while avoiding any theoretical concerns associated with aluminium use and accumulation in long-course therapy [5].

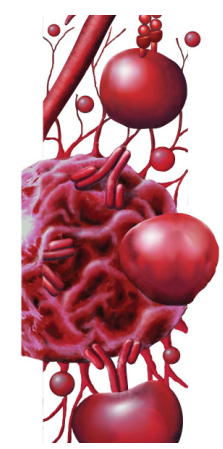

Albert Roger ${ }^{1}$, Nathalie Depreux ${ }^{1}$, Yanina Jurgens', Aina T Serra', Matthew D Heath*,2, Gloria Garcia ${ }^{3}$ \& Murray A Skinner ${ }^{2}$ 'Unitat d'Al.lèrgia. Hospital Universitari Germans Trias Pujol, Badalona, Spain ${ }^{2}$ Allergy Therapeutics PIc, Dominion Way, Worthing, BN14 8SA, UK

${ }^{3}$ Allergy Therapeutics Iberica

S.L. Carrer de Joan XXIII, 15,

08950 Esplugues de Llobregat, Barcelona, Spain *Author for correspondence:

matthew.heath@allergytherapeutics.com 
The first report published by Roger et al., assessed the tolerability and safety of the dosage-increase phase (updosing; 4 weeks) of the conventional and cluster regimens of Acarovac Plus, in patients with respiratory allergy to mites under conditions of normal clinical practice [3]. A 1-year follow-up study has now been investigated, with the existing patient pool, replicating the nasal challenge tests, in vitro immunological tests, The Treatment Satisfaction Questionnaire for Medication (TSQM) and is presented herein. The efficacy was compared between the baseline and the two follow-up visits (visit one: $3-5$ weeks and visit two: 1 year).

\section{Materials \& methods}

The detailed study design, analysis and trial material are described previously [3]. Patients that were included in the initial treatment study (both with conventional [15 patients] or cluster regime [15 patients]) were selected for the 1-year follow-up visit (visit two). Institutional review board approval (Comité de Ética de Investigación Clínica [CEIC]) and extension of 1-year approval was granted with patients with informed consent.

\section{Trial design}

Patients (18-65 years old) with hypersensitivity to Dermatophagoides pteronyssinus (confirmed via specific IgE and skin-prick tests) were included in the initial treatment study. Depending on how rapidly the monthly maintenance dose is reached the regime may be referred to as either:

- Conventional regimen: the maintenance dose is reached after administering the starting doses one at a time at weekly intervals;

- Cluster or grouped regimen: more than one dose per day is administered at weekly intervals, making the initial phase shorter and so reaching the maintenance dose earlier.

The maintenance phase consists of a $0.5-\mathrm{ml}$ dose every 6 weeks.

For quantitative variables, nonparametric tests were used (Mann-Whitney and Wilcoxon), according to the particular characteristics of the study variables. For qualitative variables, a $\chi^{2}$ test or a McNemar test was used. Statistical analyses were performed with the SAS statistical package, version 9.3.

\section{Inclusion criteria}

- Patients between 18 and 65 years of age;

- Patients with allergic rhinitis and/or asthma caused by Dermatophagoides pteronyssinus for at least 1 year before taking part in the study and in whom, in the investigator's opinion, treatment with specific immunotherapy is indicated;

- Patients who have had a prick test result $\geq 3 \mathrm{~mm}$ in diameter for $D$. pteronyssinus in the previous 12 months;

- Patients may be monosensitive to Dermatophagoides or polysensitized provided that, in the investigator's opinion, the other sensitizations are not clinically relevant;

- Patients who have agreed to take part and who sign the informed consent.

Note: no patient dropouts were recorded during the course of the study.

\section{Study material}

Acarovac Plus ${ }^{\mathrm{TM}}$ contains natural aqueous extract allergens from Dermatophagoides pteronyssinus. The allergens have been modified into extracts of allergoids by treatment with glutaraldehyde and have been associated with L-Tyrosine (MCT), while undergoing a number of sterile filtration and purification steps.

The allergen extracts are characterized to ensure that allergen content and activity are constant. In the top dose vaccine, the calculated content of major allergen Der p 1 is $4.8 \mu \mathrm{g} / \mathrm{ml}$. Its pharmaceutical form is a white, opaque suspension for subcutaneous injection.

\section{Nasal provocation test}

The Nasal Provocation Test (NPT) was carried out measuring peak nasal inspiratory flows (PNIF)s in the baseline visit (V0) and the two follow-up visits (V1; 3-5 weeks and V2; 1 year) in response to controlled exposure to the allergen. In visits two to three, NPT was carried out using the allergen concentration with which the test was positive at the baseline visit. A positive response was considered as: five or more sneezes or a drop in the peak nasal inspiratory flow greater than $50 \%$ (the highest value is always taken to calculate the percent of the drop in peak nasal inspiratory flow). The methodology followed was according to SEAIC guidelines [6] and was described previously [3].

Symptom scores (0-9) are based on the sum of the three symptoms (sneezing, secretion and nasal itching).

\section{In vitro immunological measurements}

Detection of immunoglobulins in serum was performed using ELISA ${ }^{\mathrm{TM}}$ (ImmunoCAP ${ }^{\circledR}$; Phadia AB Uppsal, Sweden) using the $250^{\circledR}$ analyzer Immuno- 


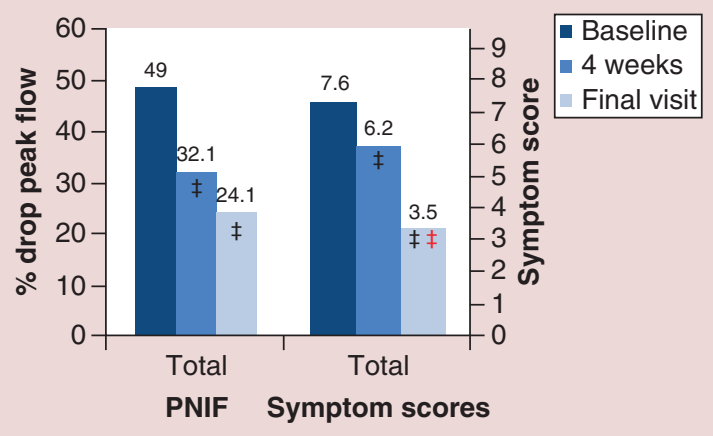

Figure 1. Patient nasal inspiratory flow and symptom scores at baseline and follow-up visits. Symptom scores (0-9) are based on the sum of the three symptoms (sneezing, secretion and nasal itching).

₹ Statistically different results were observed (Wilcoxon test compared with baseline data; $p<0.05$ ).

$\ddagger($ red) Statistically different results were observed (Wilcoxon test compared with 4-week data; $p<0.05$ ).

PNIF: Patient nasal inspiratory flow.

CAP (Phadia AB). Cytokine levels in serum were measured by Cytometric bead array (Becton Dickinson Biosciences, CA, USA). Samples were acquired on a flow cytometer (LSR Fortessa, BD Biosciences).

Note: results are expressed as $\mathrm{mgA} / 1$ (measuring range: $0.07-30 \mathrm{mgA} / \mathrm{l})$.

\section{TSQM questionnaire}

TSQM has been validated in a study by Atkinson et al. [7]. In the present study TSQM was performed during follow-up visits (V2 and V3).

\section{Results}

Symptoms scores decreased 18.6 and $54.9 \%$ in V1 and V2, respectively. This was significant (Wilcoxon test; $\mathrm{p}<0.05)$ at both visits relative to baseline and also at the final visit compared with visit two (Figure 1). The decrease in the drop of PNIF was 34.5 and $50.8 \%$ at $\mathrm{V} 1$ and $\mathrm{V} 2$, respectively. This decrease was statistically significant (Wilcoxon test; $\mathrm{p}<0.05$ ) at both follow-up visits compared with baseline, but not at the final visit compared with visit two (Figure 1).

Statistically significant increases were observed for specific IgG4 at the two follow-up visits compared with the baseline visit (Wilcoxon test; $\mathrm{p}<0.05$ ), Figure 2. Note: differences in total and specific IgE between the follow-up visits compared with the baseline visit were not statistically significant (Wilcoxon test; $\mathrm{p}<0.05$ ) (data not shown).

Statistically significant increases compared with the baseline visit were observed IL-10 at the final visit (V2) (Wilcoxon test; $\mathrm{p}<0.05$ ), Figure 3.

TSQM followed a similar suit as described in Roger et al. [3]. Of note, a significant improvement was in relation to 'effectiveness,' resulting in a significant 38\% improvement. Overall, the average total score for the TSQM questionnaire was 66.7 (standard deviation $=11.5$ ) and 76.2 (standard deviation $=12.4)$, respectively (Figure 4$)$.

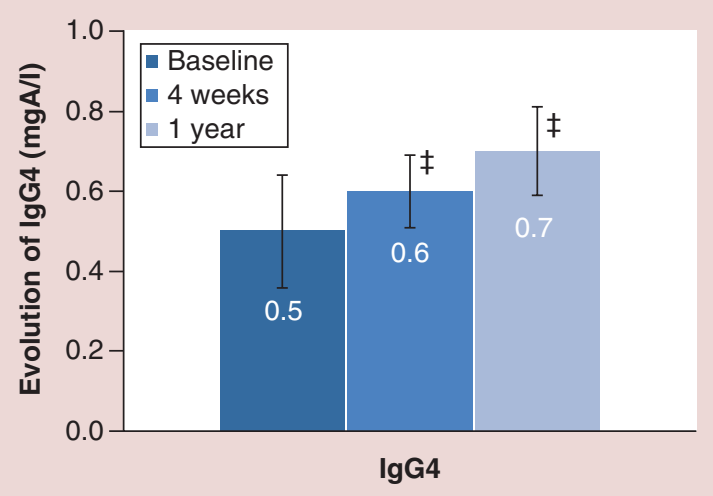

Figure 2. Evolution of IgG4 at baseline and follow-up visits. Results are expressed as mgA/l (measuring range: $0.07-30 \mathrm{mgA} / \mathrm{l}$ ).

‡ Statistically significant differences were observed (Wilcoxon test compared with baseline data; $p<0.05$ ). 


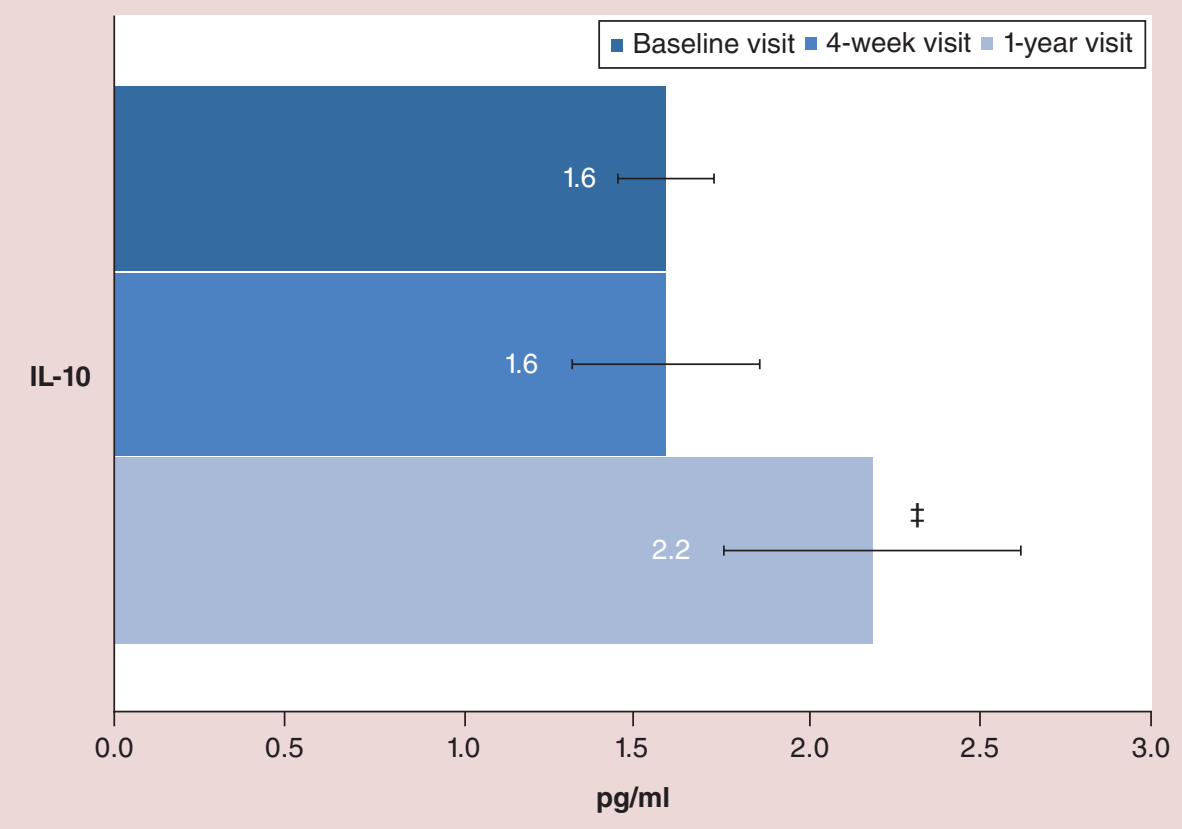

Figure 3. Evolution of cytokine (IL-10) at baseline and follow-up visits. IL-10 units are expressed pg/ml. ¥ Statistically significant differences were observed compared with baseline data (Wilcoxon test; $p<0.05$ ).

\section{Discussion}

The results herein described observational data using the current marketed product - Acarovac Plus $^{\mathrm{TM}}$. The assessment of clinical symptoms (score) and drop in PNIF were first compared by measuring responses (from baseline and follow-up visits) of the nasal mucosa after controlled exposure to the allergens (NPT), followed by the assessment of immunological parameters and patient satisfaction. The results demonstrated marked and, in some cases, significant improvements occurring in each assessment.

The clinical efficacy of specific immunotherapy treatment is well documented and, in particular, the comparison between clinical efficacy and immunological parameters has been widely pre-

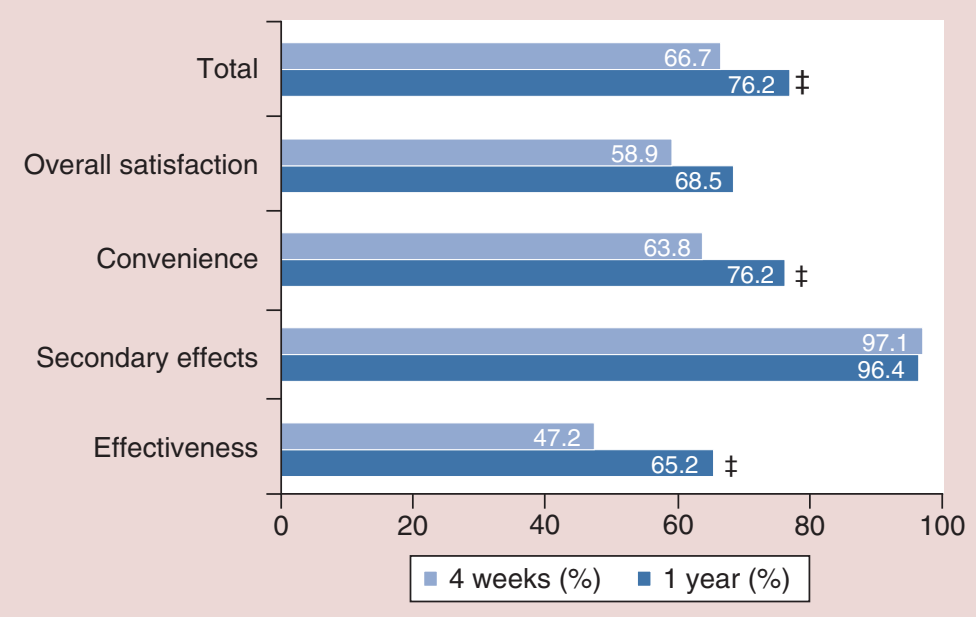

Figure 4. The total average categorized The Treatment Satisfaction Questionnaire for Medication question scores (\%). Range: $0-100 ; 0=$ not at all; $100=$ very strong.

‡ Statistically significant differences were observed compared with data from the 4-week visit (Wilcoxon test; $\mathrm{p}<0.05)$. 
sented across different treatments for different allergies [8-12]. More specifically, a number of key studies have demonstrated longer-term clinical efficacy of SCIT treatment for patients with house dust mite allergy [13,14]. Such studies have demonstrated similar reductions in patient symptom scores and immunological parameters (e.g., significant increase in IgG4 and IL-10 production after 1-2 years).

While it is difficult to ascertain the significance of these results in the absence of a placebo arm, the current marketed formulation provides assurances on safety tolerability in the first instance, highlighting changes in in vitro immunologic characteristics associated with desensitization in adult patients with allergic rhinitis and/or asthma.

No other studies currently exist using an allergoid formulation combined with a biodegradable depot adjuvant for specific mite subcutaneous immunotherapy. The use of new and alternative biodegradable adjuvants is encouraged [15]. Studies to address the long-term efficacy in a dbpc setting based on the current marketed product, Acarovac Plus ${ }^{\mathrm{TM}}$, are now in development.

\section{Conclusion}

Significant decreases in clinical symptoms and immunological parameters were observed, accompanying a high level of patient satisfaction and tolerance.

\section{Financial \& competing interests disclosure}

A Roger has received fees as a speaker, principal investigator of the study and consultant of Allergy Therapeutics Plc. At the time of writing MD Heath, G Garcia, MA Skinner are employees of Allergy Therapeutics. The authors have no other relevant affiliations or financial involvement with any organization or entity with a financial interest in or financial conflict with the subject matter or materials discussed in the manuscript apart from those disclosed.

No writing assistance was utilized in the production of this manuscript.

\section{Ethical conduct of research}

The authors state that they have obtained appropriate institutional review board approval or have followed the principles outlined in the Declaration of Helsinki for all human or animal experimental investigations. In addition, for investigations involving human subjects, informed consent has been obtained from the participants involved.

\section{Executive summary}

- Acarovac Plus ${ }^{\top \mathrm{M}}$ was well tolerated and no adverse events were reported during dosing schedules in this study.

- Significant decreases in symptom scores and drop of peak nasal inspiratory flow in both follow-up visits (V1; 4 weeks and V2; 1 year) were recorded.

- Significant increases in IgG4-specific antibody titers were observed during both follow-up visits, with a significant increase in IL-10 exhibited after 1 year.

- Patients expressed a high level of patient satisfaction (The Treatment Satisfaction Questionnaire for Medication).

\section{References}

1 Sala-Cunill A, Bartra J, Dalmau G et al. Prevalence of asthma and severity of allergic rhinitis comparing 2 perennial allergens: house dust mites and Parietaria judaica pollen. J. Investig. Allergol. Clin. Immunol. 23(3), 145-151 (2013).

2 Calderon MA, Linneberg A, Kleine-Tebbe J et al. Respiratory allergy caused by house dust mites: what do we really know? J. Allergy Clin. Immunol. 136(1), 38-48 (2015).

3 Roger A, Depreux M, Jurgens Y et al. A novel and well tolerated mite allergoid subcutaneous immunotherapy: evidence of clinical and immunologic efficacy. Immun. Inflam. Dis. 2, 92-98 (2014).

4 Jongejan L, van Ree R. Modified allergens and their potential to treat allergic disease. Curr. Allergy Asthma Rep. 14(12), 478 (2014).

5 Kramer MF, Heath MD. Aluminium in allergen-specific subcutaneous immunotherapy: a German perspective. Vaccine 32(33), 4140-4148 (2014).

6 Dordal MT, Lluch-Bernal M, Sánchez MC et al. Allergenspecific Nasal Provocation Testing: review by the Rhinoconjunctivitis Committee of the Spanish Society of
Allergy and Clinical Immunotherapy. J. Investig. Allergol. Clin. Immunol. 21(1), 1-12 (2011).

7 Atkinson MJ, Sinha A, Hass SL et al. Validation of a general measure of treatment satisfaction, The Treatment Satisfaction Questionnaire for Medication (TSQM), using a national panel study of chronic disease. Health Qual. Life Outcomes 26(2), 12 (2004).

8 Eifan T, Akkoc A, Yildiz S et al. Clinical efficacy and immunological mechanisms of sublingual and subcutaneous immunotherapy in asthmatic/rhinitis children sensitized to house dust mite: an open randomized controlled trial. Clin. Exp. Allergy 40, 922-932 (2010).

9 Khinchi MS, Poulsen LK, Carat F et al. Clinical efficacy of sublingual and subcutaneous birch pollen allergenspecific immunotherapy: a randomized, placebo-controlled, double-blind, double-dummy study. Allergy 59, 45-53 (2004).

10 Mungan D, Misirligil Z, Gurbuz L. Comparison of the efficacy of subcutaneous and sublingual immunotherapy in mite-sensitive patients with rhinitis and asthma - a placebo controlled study. Ann. Allergy Asthma Immunol. 82, 485-490 (1999). 
11 Tahamiler R, Saritzali G, Canakcioglu S et al. Comparison of the long-term efficacy of subcutaneous and sublingual immunotherapies in perennial rhinitis. J. Otorhinolaryngol. Relat. Spec. 70, 144-150 (2008).

12 Antu'nez C, Mayorga C, Corzo JL et al. Two year follow-up of immunological response in mite-allergic children treated with sublingual immunotherapy. comparison with subcutaneous administration. Pediatr. Allergy Immunol. 19, 210-218 (2008).

13 Olsen OT, Larsen KR, Jacobsen L, Svendsen UG. A 1-year, placebo controlled, double-blind house-dust-mite immunotherapy study in asthmatic adults. Allergy 52 , 853-859 (1997).

14 Yekselen A, Kendirli SG, Yilmaz M, Altinas DU, Karakoc GB. Two year follow-up of clinical and inflammation parameters in children monosensitized to mites undergoing subcutaneous and sublingual immunotherapy. Asian Pac.J. Allergy Immunol. 31, 233-241 (2013).

15 Jensen-Jarolim E. Aluminium in allergies and allergen immunotherapy. World Allergy Organ. J. 8, 7 (2015). 